ISSN 2447-9071

doi https://doi.org/10.36414/rbmc.v7i18.100

Contato para correspondência:

Clayson Moura Gomes

E-mail:

claysonmoura@yahoo.com.br

Conflito de interesse: Não

Financiamento: Recursos próprios

Recebido: $24 / 08 / 2021$

Aprovado: 25/08/2021

\section{CARACTERIZAÇÃO DE ESPERMOGRAMAS REALIZADOS EM POLICIAIS MILITARES ATENDIDOS NO LABORATÓRIO DO HOSPITAL DO POLICIAL MILITAR DE GOIÁS}

\section{Characterization of spermograms performed on military po- lice officers served in the laboratory of the military police hos- pital of Goiás}

Ludmila Felipe Oliveira', Letícia Romeira Belchior ${ }^{2}$, Sérgio Henrique Nascente Costa ${ }^{1,2}$ Clayson Moura Gomes ${ }^{1,2}$

\author{
1 Faculdade da Polícia Militar - FPM \\ 2 Pontifícia Universidade Católica de Goiás - PUC Goiás
}

\begin{abstract}
Resumo
A importância da avaliação da saúde sexual do homem é relevante e contribui para prevenção e cuidado com relação a doenças e causas de disfunções sexuais ou infertilidade. $\mathrm{O}$ espermograma é um exame importante para verificar se o volume do esperma, o pH (acidez), a viscosidade, a cor e a liquefação do sêmen apresentam-se normais, dentre outras análises. A caraterização do espermograma de determinados homens, como os militares, contribui para uma abordagem comparativa com a literatura e detecção de associações com hábitos de vida específicos que auxiliam na prevenção e cuidados essenciais para a melhoria da saúde. O objetivo deste estudo é de caracterizar os espermogramas em policiais militares atendidos no laboratório do Hospital do Policial Militar de Goiás e relacionar com fatores antropométricos e metabólicos. Trata-se de um estudo transversal realizado entre os anos de 2008 e 2018, em que foram descartados os pacientes vasectomizados. Assim, os resultados de 46 espermogramas, no período de 2008 a 2018 foram tabulados e associados com resultados de glicemia em jejum, perfil lipídico e IMC. Os resultados de três pacientes vasectomizados foram excluídos, sendo que nos 43 indivíduos restantes, a média de idade foi de 36 anos e 70\% (9/13) apresentaram valores aumentados do IMC. Com relação aos níveis glicêmicos, 18\% (3/17) manifestaramse elevados e observou-se quadro de dislipidemias em 40\% (6/15) dos analisados. Em relação as alterações encontradas no espermograma: $37 \%$ possuíam necrospermia; 5\% apresentaram teratospermia; $37 \%$ dos indivíduos apontam hipospermia; $24 \%$ manifestaram oligospermia; $50 \%$ dos avaliados apontam astenopermia. A caracterização dos espermogramas, juntamente com fatores antropométricos e metabólicos dos policiais militares, mostraram que é importante o rastreio e as pesquisas relacionadas a esse assunto, pois podem estar relacionados com a fertilidade e hábitos importantes na vida desses profissionais.
\end{abstract}

Palavras-chave: Fertilidade, espermograma, reprodução humana, militares.

\begin{abstract}
The importance of evaluating men's sexual health is relevant and contributes to prevention and care in relation to diseases and causes of sexual dysfunctions or infertility. Spermogram is an important test to verify that sperm volume, $\mathrm{pH}$ (acidity), viscosity, color and liquefaction of semen are normal, among other analyses. The characterization of the spermogram of certain men, such as the military, contributes to a comparative approach with the literature and detection of associations with specific life habits that help in the prevention and care essential for the improvement of health. The aim was characterize spermograms in military police officers served in the laboratory of the Military Police Hospital of Goiás and relate to sociodemographic factors. This is a cross-sectional study conducted between 2008 and 2018. Forty-three spermograms were analyzed, and
\end{abstract}


vasectomized were discarded from the study, and associated with fasting blood glucose, lipid profile and BMI results. A total of 43 men were analyzed between 2008 and 2018, and 3 were vasectomized, thus, among the 40 individuals had an average of 36 years, $70 \%(9 / 13)$ had increased BMI values. Regarding glycemic levels $18 \%(3 / 17)$ showed high, observed in the lipid profile elevation in $40 \%(6 / 15)$ of the analyzed. Data related to alterations found in the spermogram: $37 \%$ have necrospermia; $5 \%$ have teratospermia; $37 \%$ of the individuals point hypospermia; $24 \%$ manifest oligospermia; $50 \%$ of the evaluated point astenopermia. The characterization of spermograms together with sociodemographic factors of the military police show that screening and research related to this subject is important, because they can be related to fertility and important habits in the lives of these professionals.

Keywords: Fertility, spermogram, human reproduction, military.

\section{Introdução}

A infertilidade é considerada pela Organização Mundial de Saúde (OMS), a incapacidade de conceber um filho após 12 meses de relação sexual regular sem o uso de contraceptivos. Sendo que, na grande maioria, apenas um dos parceiros seja diagnosticado com a infertilidade. Estudo aponta que há um menosprezo de pesquisas da infertilidade em homens, focando mais em investigações em mulheres. Portanto, a atitude de ignorar as doenças potencialmente curáveis dos homens é muito prevalente. Desta forma, uma avaliação preliminar completa deve ser realizada para que as doenças potencialmente curáveis possam ser diagnosticadas e tratadas adequadamente. Sendo essencial na investigação do homem infértil, um histórico detalhado e um exame físico completo e laboratorial'.

O espermograma é um exame laboratorial de grande importância para verificar a saúde da próstata e dos espermatozoides produzidos pelo homem, através de uma amostra de sêmen coletada por via da masturbação, seguindo as orientações da OMS. Sendo averiguado nesta análise, o volume do esperma, o pH (acidez), a viscosidade, a cor, concentração de espermatozoides, a liquefação do sêmen (capacidade de se tornar líquido), a velocidade de progressão dos espermatozoides, o movimento (ou motilidade) dos espermatozoides, dentre outras análises².

A caraterização do espermograma de determinados homens, como os militares, contribui para uma abordagem comparativa com a literatura e detecção de associações com hábitos de vida específicos, que auxiliam na prevenção e cuidados essenciais para a melhoria da saúde. Tendo em vista que, fatores como estresse podem causar vários transtornos no bem-estar físico, funcional e emocional para o organismo dos seres humanos. Ao avaliar o estilo de vida dos militares, é notório que, altas cargas horárias e a grande responsabilidade de exercer essa função, fazem com que os policiais se estressem e deixem de lado hábitos saudáveis, adotando, consequentemente, perfis sedentários e má alimentação³.
Diante desse contexto, a má qualidade de vida dos militares pode acarretar transtornos diversos, como a infertilidade. Visto que, o estresse pode causar o aumento de peso, gerando obesidade, que reduz a qualidade do esperma e a concentração da testosterona. Desta forma, é de grande relevância um estudo que caracterize os espermogramas na população militar, para que danos futuros na saúde reprodutiva dessa população sejam evitados ${ }^{4}$. O objetivo deste estudo é caracterizar espermogramas em policiais militares atendidos no laboratório do Hospital do Policial Militar de Goiás e relacionar com fatores sociodemográficos.

\section{Métodos}

\section{Tipo de Estudo}

Trata- se de um estudo transversal, no qual foi efetuado uma tabulação e análise de dados no banco de dados do laboratório do Hospital do Policial Militar de Goiás. Foram extraídos resultados dos indivíduos que realizaram o exame do espermograma e outros como glicemia, lipidograma, colesterol, triglicérides e testosterona, no período de janeiro do ano de 2008 até dezembro de 2018. Foram considerados todos os homens que realizaram espermograma nesse período, destes, foram descartados do estudo os indivíduos vasectomizados, totalizando assim um $\mathrm{n}$ de 43.

\section{Considerações Éticas}

Os dados extraídos tiveram sua confidencialidade resguardada, obtendo ao final resultados em dados estatísticos. O trabalho foi submetido à plataforma Brasil, ao CEP da PUC Goiás sob parecer de número 608.207 de 24/03/2014 e atende às exigências da Resolução 466/12.

\section{Análise Estatística}

Para realização da análise descritiva foi calculada a média, mediana, frequência absoluta e percentual, além do desvio padrão das variáveis, quando aplicável. Foi utilizado o software 
Microsoft Excel versão 2016 ou o GraphPad versão 6.0 para a análise e construção de tabelas e gráficos.

\section{Resultados}

Um total de 43 amostras de policiais militares foram analisadas laboratorialmente. A coleta de dados foi realizada entre os anos de 2008 e 2018, de uma amostra toda do sexo masculino e com idade média de 36 anos.

No parcial de sujeitos, foi verificado que $70 \%(9 / 13)$ apresentaram valores aumentados do Índice de Massa Corpórea (IMC). Com relação aos níveis glicêmicos 18\% (3/17) manifestaram-se elevados, levando em consideração o valor referencial. Foi observado no perfil lipídico elevação em 40\% (6/15) dos indivíduos analisados. Em relação ao HDL, 27\% (4/15) dos resultados encontram-se com índices aumentados, estando fora dos parâmetros referenciais. Outros exames avaliados como triglicérides tiveram 33\% (5/15) com índices aumentados e testosterona, $11 \%$ (2/18) encontraram-se aumentados e $6 \%$ $(1 / 18)$ dos indivíduos apresentaram valores abaixo do valor de referência (Tabela 1).

Tabela 1: Características Epidemiológicas e Laboratoriais dos Participantes

\begin{tabular}{ccccc}
\hline Variável & Normal $^{*}$ & $\begin{array}{c}\text { Aumen- } \\
\text { tado* }\end{array}$ & $\begin{array}{c}\text { Diminu- } \\
\text { ído* }\end{array}$ & Média \\
\cline { 1 - 1 } Idade (a) & & & & 36 \\
Altura (m) & & & & 1,75 \\
Peso (kg) & & & & 84 \\
IMC & $30 \%$ & $70 \%$ & $0 \%$ & \\
Glicemia & $82 \%$ & $18 \%$ & $0 \%$ & \\
Colesterol Total & $60 \%$ & $40 \%$ & $0 \%$ & \\
HDL & $73 \%$ & $0 \%$ & $27 \%$ & \\
Triglicérides & $67 \%$ & $33 \%$ & $0 \%$ & \\
Testosterona & $83 \%$ & $11 \%$ & $6 \%$ & \\
\hline
\end{tabular}

Legenda: IMC: Índice de Massa Córporea; HDL(High Density Lipoproteins, Lipoproteínas de alta densidade). *Em relação aos valores de referência. a:anos; m:metros; kg: kilogramas

No total de sujeitos analisados, a média de abstinência sexual foi de 4 dias. Com relação ao volume do ejaculado das amostras, $63 \%$ apresentam volumes normais e $37 \%$ alterados. Os índices de liquefação estavam normais em $82 \%$ e baixos em $18 \%$, sendo assim, a pós liquefação apresenta $79 \%$ normal e $21 \%$ abaixo. No levantamento dos níveis de $\mathrm{pH}, 71 \%$ encontraram-se dos exames se encontraram com níveis normais e $29 \%$ aumentados. Em relação à viscosidade, $71 \%$ dos participantes apresentaram níveis normais e $29 \%$ elevados. Dentro desta caracterização, 76\% obtiveram níveis normais de espermatozoides e $24 \%$ níveis baixos. Com relação às células sanguíneas encontradas nos espermogramas, sendo elas, leucócitos e eritrócitos apresentaram 95\% com quantidades normais e $5 \%$ aumentados. Dentro do levantamento da caracterização dos espermatozoides (SPTZ), 84\% obtiveram normalidade para sua motilidade e $16 \%$ baixa motilidade, $74 \%$ obtiveram níveis normais para SPTZ rápidos e 26\% diminuídos, sendo que, $32 \%$ apresentaram aumentados para progressão lenta e 68\% encontram-se normais. Espermatozoides imóveis: 82\% normais e $18 \%$ elevados. Eosina negativa: $63 \%$ normais e $37 \%$ baixas, com relação a SPTZ anormais: $95 \%$ normais e $5 \%$ com alteração (Tabela 2). Os resultados do espermograma foram interpretados de acordo com os critérios da Organização Mundial da Saúde (OMS)².

Tabela 2. Características Laboratoriais dos Espermogramas dos Participantes

\begin{tabular}{cccc}
\hline Características & Normal* & $\begin{array}{c}\text { Aumen- } \\
\text { tado* }\end{array}$ & $\begin{array}{c}\text { Diminu- } \\
\text { ído* }\end{array}$ \\
\hline Volume & $63 \%$ & $0 \%$ & $37 \%$ \\
Pós Liquefação & $79 \%$ & $21 \%$ & $0 \%$ \\
Liquefação & $82 \%$ & $0 \%$ & $18 \%$ \\
pH & $71 \%$ & $29 \%$ & $0 \%$ \\
Viscosidade & $71 \%$ & $29 \%$ & $0 \%$ \\
№ espermatozoide & $76 \%$ & $0 \%$ & $24 \%$ \\
Leucócitos & $95 \%$ & $5 \%$ & $0 \%$ \\
Eritrócito & $95 \%$ & $5 \%$ & $0 \%$ \\
Móveis & $84 \%$ & $0 \%$ & $16 \%$ \\
Rápidos & $74 \%$ & $0 \%$ & $26 \%$ \\
Lentos & $68 \%$ & $32 \%$ & $0 \%$ \\
Imóveis & $82 \%$ & $18 \%$ & $0 \%$ \\
Eosina negativa & $63 \%$ & $0 \%$ & $37 \%$ \\
Anormais & $95 \%$ & $5 \%$ & 0 \\
\hline
\end{tabular}

Legenda: *De acordo com os valores de referência; №: Número. Média de Abstinência para o exame: 4 dias.

Dentre as avaliações realizadas neste estudo, e visível que, $37 \%$ possuem necrospermia, que é o termo utilizado para a condição de espermatozoides mortos no sêmen. Nos espermogramas de policiais militares, participantes deste estudo analisados, $5 \%$ apresentam teratospermia que é uma condição na qual, a morfologia dos espermatozoides é anormal, presentes no sêmen ou ejaculado. Já $37 \%$ dos indivíduos apontam hipospermia, caracterizada pelo volume de sêmen ejaculado abaixo de 1,5 mL. A oligospermia (concentração de espermatozoides abaixo de 15 milhões $/ \mathrm{ml}$ ) foi manifestada em $24 \%$ dos analisados (Figura 1). Já 50\% dos avaliados apresentaram 
astenopermia, uma condição em que os espermatozoides têm uma motilidade reduzida, sendo eles lentos ou imóveis².

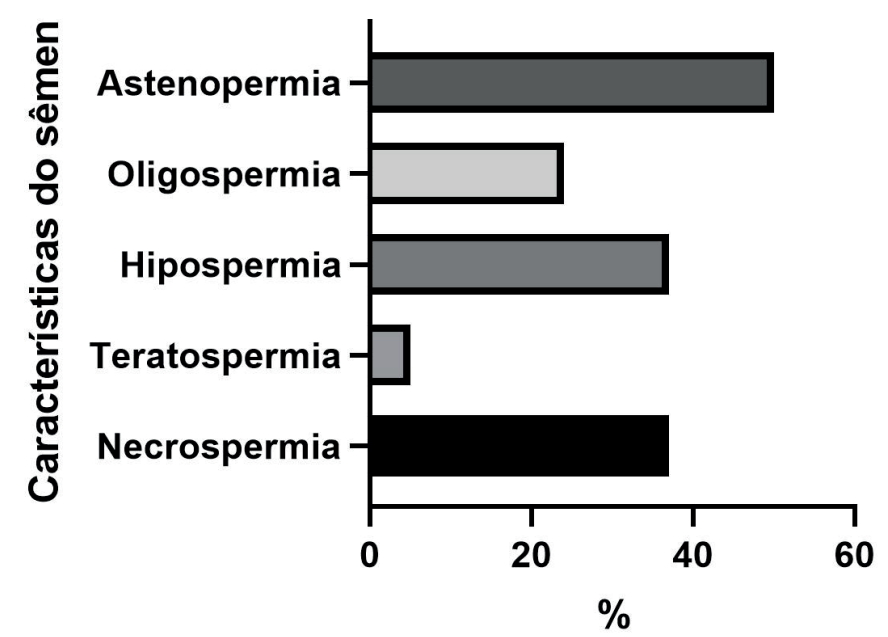

Figura 1. Caracterização do sêmen nos espermogramas dos participantes. \% Porcentagem do total.

\section{Discussão}

Pressupõe-se que a infertilidade atinge até 186 milhões de indivíduos no mundo. Ademais, deve ser considerado que, os homens contribuem para mais da metade dos casos, contudo, as mulheres continuam sendo apontadas como as principais responsáveis da infecundidade pela sociedade ${ }^{6,7}$. Sendo que, uma análise citométrica revelou que o aumento da idade do indivíduo relaciona-se com os distúrbios funcionais dos espermatozoides, tanto no reconhecimento e penetração (acrossoma), quanto na energia da célula e na densidade da cromatina em seu núcleo ${ }^{8}$.

Com relação à esterilidade masculina, há vários fatores que podem contribuir para que isso ocorra, sendo um deles, o estilo de vida, analisado nesse estudo com os policiais militares do estado de Goiás. O estilo de vida inadequado tem efeitos destrutivos na qualidade do esperma e na fertilidade masculina, de modo que a modificação do estilo de vida pode melhorar os dados preliminares dos índices do espermograma. Esta pesquisa aponta que há maior prevalência de sobrepeso nos policiais quando são submetidos à avaliação do IMC. Levando em consideração que, o estilo de vida desses profissionais é de elevada carga horária de trabalho e de grande responsabilidade em exercer sua função, o estresse é uma consequência, conjuntamente ao abandono de hábitos saudáveis. Assim, passam a ser sedentários e adquirem uma má alimentação, o que pode afetar a fertilidade ${ }^{3,5,6}$.

Considera-se hipospermia quando o volume de sêmen ejaculado é abaixo de 2,0 mL. Existem muitas razões para que ocorram hipospermias, que podem ser divididas em dois grupos fisiopatológicos, o primeiro é o reflexo da ejaculação anormal, levando à ejaculação retrógrada parcial e danos anatômicos e funcionais às glândulas e ao trato seminal. $\mathrm{O}$ tratamento da causa da hipospermia deve ser considerado e realizado o mais rápido possível, se não pode trazer danos a fertilidade do paciente posteriormente ${ }^{7,10}$. É importante ressaltar que $37 \%$ dos policiais analisados neste estudo apresentaram hipospermia. Sendo assim, é de suma importância o diagnóstico precoce para evitar o comprometimento da fertilidade desses profissionais.

Já a oligospermia está ligada a $24 \%$ dos participantes analisados no estudo e como geralmente não está associada a distúrbios gerais ou locais, os pacientes não procuram atendimento médico ou, após vários anos de casamento, consideram desnecessário investigar sua possível infertilidade. A oligospermia pode ser motivada por várias causas, incluindo hábitos prejudiciais aos testículos. Isso pode variar de roupas justas a alimentos e hábitos como tabagismo e alcoolismo. Outro fator é o estresse diário, porque também pode reduzir a quantidade e a qualidade dos espermatozoides, causando oligospermia transitória, que é completamente reversível ${ }^{11,12}$. Levando em consideração a rotina estressante dos policiais militares, seu fardamento quente e a má alimentação, a oligospermia pode estar ocorrendo por esse motivo, sendo necessário ficar em alerta com esses hábitos estressantes cotidianos, pois podem trazer danos à saúde reprodutiva.

Outrossim, a astenopermia é estimada em média 50\% dos policiais em nosso estudo, o que é extremamente preocupante, esses profissionais precisam buscar um médico no primeiro sinal de alteração de fertilidade e realizarem um espermograma, isso porque, há dificuldade na motilidade espermática durante o transporte epididimal. A movimentação do esperma é muito importante para a sua migração na vagina para a trompa de Falópio, penetração e processo de fertilização. Portanto, há uma correlação entre a motilidade dos espermatozoides e a chance de concepção natural. Porém é notório e relatado na literatura a rejeição dos homens para realizarem consultas e tratamentos, um problema que é agravado quando se trata de exames mais íntimos, como o espermograma. O que é explícito no estudo em questão, no qual, metade dos analisados possuem astenopermia, que pode ser relacionada com a vergonha de realizar o exame $e^{7,13,15}$.

A contagem de espermatozoides aumentada ou dentro dos valores de referência, não é sinônimo de boa qualidade, quando avaliada separadamente; na verdade, pode parecer que o esperma contém espermatozoides suficientes, porém, mais da metade pode estar morta após a coloração com eosina ou um teste de edema hipoosmótico. Tendo em vista que, 37\% 
dos nossos analisados sofrem de necrosospermia, a origem desse acontecimento pode ser testicular (hipertireoidismo, varicocele, hipertermia), pos-testicular (necrospermia epididimal, anormalidades do plasma do esperma, doença renal policística, pós-vasostomia, anticorpos anti-espermatozoides) ou mistos (infecção, tóxico, idade, lesão da medula espinhal). 0 tratamento é principalmente etiológico, sempre que possível. As taxas de fertilização são baixas em casos de necrospermia, mas as técnicas de fertilização in vitro com microinjeção intracitoplasmática de espermatozoides aumentam as chances de concepção ${ }^{14,7}$.

Cerca de $40 \%$ da frequência da infertilidade é causada por anormalidades masculinas. Metade dos casos de infertilidade masculina são causados por baixa motilidade espermática (astenopermia), contagem diminuída de espermatozoides (oligospermia) ou morfologia espermática anormal (teratozoospermia). Segundo a OMS, o formato normal e perfeito dos espermatozoides deve ter cabeça, corpo e cauda. Muitos fatores podem fazer com que a produtividade dos homens caia, não tendo assim um bom ambiente para os espermatozoides, como por exemplo, um estilo de vida baseado em uma dieta alimentar ou hábitos diários prejudiciais à saúde, o que além disso, podem piorar a qualidade do esperma. $\mathrm{O}$ laboratório ainda se baseia na morfologia dos espermatozoides para formar um modelo de observação da reprodução espermáticaa ${ }^{15,16,2}$. Tendo em vista que, $5 \%$ dos militares analisados apresentaram teratospermia, novamente é importante ressaltar a relevância da qualidade de vida desses profissionais, a qual é refletida diretamente na saúde reprodutora e afeta consequentemente as esferas biopsicossociais da saúde ${ }^{17}$. Isso porque, a possibilidade de não ter filhos traz consigo um peso individual, dentro do ambiente familiar e comunitário.

Com relação aos parâmetros epidemiológicos e laboratoriais dos participantes que realizaram o espermograma, é notório que o índice de massa corpórea (IMC) encontra-se com grandes taxas de alteração, acometendo 70\% (9/13) dos pacientes que realizaram espermograma no mesmo período, sendo correlacionados também com índices do perfil lipídico que se apresentam elevado em $40 \%$ (6/15) dos analisados, além disso, outro fator que não está dentro dos parâmetros analisados é a glicemia, a qual encontra-se elevada em $18 \%$ (3/17) dos indivíduos.

Por conseguinte, o aumento do IMC, lipidograma e da glicemia estão correlacionados como um fator prejudicial à saúde do homem e sua fertilidade. Pode-se observar que, em comparação com homens com peso normal, homens com sobrepeso e obesos têm maior probabilidade de terem alteração na concentração de esperma, motilidade, viabilidade, morfologia, integridade do DNA, função mitocondrial, diminuição dos hormônios sexuais e aumento da leptina e baixo nível de testosterona ${ }^{18,19}$. Com base no que foi apresentado, $\mathrm{O}$ estilo de vida dos policiais militares do estado de Goiás, pode afetar sua fertilidade. Desta forma, mecanismos devem ser criados para que isso não ocorra nessa corporação. São imprescindíveis mais informações sobre o espermograma, para que possam ter um diagnóstico precoce de possíveis acometimentos patológicos e mais estudos científicos complementares nesta área.

\section{Conclusão}

Com base no que foi analisado, a caracterização dos espermogramas juntamente com a análise do perfil sociodemográfico dos policiais militares, mostram que são importantes o rastreio e pesquisas sobre esse assunto, porque podem estar relacionadas com a fertilidade e hábitos importantes na vida desses profissionais.

Dessa maneira, foi evidenciado que um estilo de vida não saudável correlacionado com fatores estressantes da rotina dos policiais do grupo amostral estudado, podem afetar diretamente na saúde do esperma e na integralidade biopsicossocial do indivíduo.

\section{Referências}

1. Gameiro, S, Silva S, Canavarro MC. A experiência masculina de infertilidade e de reprodução medicamente assistida. Psicologia, Saúde \& Doenças. 2008; 9(2): 253-270.

2. Organização Mundial da Saúde. Manual de laboratório da Organização Mundial da Saúde (OMS) para o exame e processamento de sêmen humano. 2010; (5). Acesso em: 23 de Julho de 2021. Disponível em: https://www. who.int/reproductivehealth/publications/infertility.

3. Oliveira, KL, Santos, LM. Percepção da saúde mental em policiais militares da força tática e de rua. Sociologias. 2010; 12(25).

4. Farias, LT, De Sabóia, RCB, Lima CHR. Obesidade e infertilidade: uma revisão sistemática da literatura. Revista Interdisciplinar. 2018; 10(3):141-149.

5. Reis-Junior, J. Avaliação da composição corporal em policiais militares do $22^{\circ}$ batalhão da Polícia Militar do Estado de Goiás. 2009.

6. Amini L, Kahrobaie M, Amiri-Farahani L, Haghani H. The relationship between health life style and spermogram Indicators among infertile men: preliminary data. BMC Res Notes. 2020;13(1):278.

7. Zemrani Y, Taoufik L, Ait Zirri K, Ghoundale O, El 
Mezouari E and Moutaj R. Spermogram and Male Fertility: Hospital Experience in Military Avicenna of Marrakech. American Journal of Medical Case Reports. 2019; 7(6):104-108.

8. Inhorn MC, Patrizio P. Infertility around the globe: new thinking on gender, reproductive technologies and global movements in the 21st century. Hum Reprod Update 2015; 21:411-26.

9. Dotsenko AA, Serebriakova MK, Kudryavtsev IV, Polevshchikov AV. Adv Gerontol. 2021;34(1):96-101.

10. Robin G, Marcelli F, Mitchell V, Marchetti C, Lemaitre L, Dewailly D, Leroy-Billiard M, Rigot J.-M. Pourquoi et comment réaliser un bilan d'hypospermie? Gynécologie Obstétrique \& Fertilité. 2008; 36(10): 1035-1042.

11. González, RR. Algunas observaciones al empleo del tapón de Weisman en la oligospermia. Revista Colombiana de Obstetricia y Ginecología.1963; 14(4): 425-428.

12. McLachlan, Rl. Approach to the patient with oligozoospermia. J Clin Endocrinol Metab. 2013; 98(3):873-80.

13. Beauchamp PJ, Galle PC, Blasco L. Human sperm velocity and postinsemination cervical mucus test in the evaluation of the infertile couple. Arch Androl. 1984; 13: 107-12.

14. Dumont A, Barbotin A.-L, Lefebvre-Khalil V, Mitchell V, Rigot JM, Boitrelle F, Robin G. La necrozoospermie: du diagnostic étiologique à la prise en charge thérapeutique. Gynécologie Obstétrique Fertilité \& Sénologie. 2017; 45(4): 238-248.

15. Syahputra M, Chairani R, Rahmat, RF, Abdullah D, Napitupulu D, Setiawan MI, Andayani, U. Identificação da fertilidade masculina através da morfologia baseada em espermatozoides anormais (Teratospermia) usando o método do momento invariante. Journal of Physics. 2017; 4(2), 121-30.

16. Tamayo HS, Cardona MW. Evaluación del factor masculino mediante espermograma más práctico y económico. Rev Cubana Obstet Ginecol. 2019; 45(1):164-168.

17. Nascimento, Pedro. De quem é o problema? Os homens e a medicalização da reprodução.Saúde do homem em debate. Editora Fiocruz. 2011; 157-174.

18. Luz JDLCT, Costa LMT, Silva RA. Impacto da obesidade na fertilidade masculina. 2021. Acesso em: 23 de Julho de 2021. Disponível em: http://openrit.grupotiradentes.com:8080/xmlui/handle/set/3686.

19. Vilela SRJ, Silva RG, Santos TG, Pádua KM, Bernardes NB, Silva ABC. The Impact of Diabetes on Fertility. Revista de Psicologia.2019; 13(47): 1187-1201. 